\title{
Reduced Order and Prioritized Reference Governors for Limit Protection in Aircraft Gas Turbine Engines
}

\author{
Ye Tian* and Ilya Kolmanovsky ${ }^{\dagger \dagger}$ \\ Department of Aerospace Engineering, The University of Michigan, Ann Arbor, MI, 48109, USA
}

\begin{abstract}
This paper considers the application of the reduced order and prioritized reference governors to protect aircraft gas turbine engines against constraint violation. The aircraft gas turbine engine control system must enforce various constraints that protect the engine from surge, over-speed, over-temperature and combustion blowout conditions, while satisfying actuator magnitude and rate limits. The reference governor is an add-on scheme augmented to a nominal engine controller, that modifies set-points that the nominal controller responds to if there is a danger of constraint violation. Prioritized reference governors enforce hard constraints and soft constraints in the order of their priority in cases when strictly enforcing all the constraints is infeasible. The reduced order reference governor theory facilitates the rigorous design of reference governors based on reduced order models so that the constraints are satisfied by the full order system. Simulation results based on a gas turbine engine model are reported that illustrate constraint handling using prioritized and reduced order reference governor techniques.
\end{abstract}

\section{Nomenclature}

$\begin{array}{ll}W F E & \text { Fuel flow } \\ A J & \text { Variable area nozzle setting } \\ I G V & \text { Inlet guide vane setting } \\ O P R 1 & \text { Ratio of the HP compressor's outlet } \\ & \text { pressure to engine inlet pressure } \\ L P E M N & \text { LP compressors exit Mach number } \\ N H & \text { HP compressor's spool speed } \\ x_{i} & \text { State augmented in LQ tracking design } \\ C_{t h r u s t} & \text { The row in C matrix that corresponds to thrust output } \\ 0_{i j} & \text { Zero matrix with } i \text { rows and } j \text { columns } \\ r(t) & \text { Reference (set-point) command } \\ v(t) & \text { Reference governor output, i.e., the command actually applied to the system } \\ H, h & \text { Matrices defining inequality constraints } \\ Q & \text { Weight matrix in reference governor } \\ \tilde{O} & \text { Maximum output admissible set } \\ \tilde{v}(t) & \text { Augmented reference governor output } \\ \epsilon & \text { Slack variable } \\ \Delta_{1}, \Delta_{2} & \text { Command constraints bounds } \\ b_{i} & \text { The } i \text { th component of the command constraint matrix } \\ R_{1}, R_{2} & \text { Weight matrices used by prioritized reference governor } \\ \lambda_{i} & \text { The } i \text { th diagonal component of the weight matrix } \\ E_{x} & \text { State error set used by the reduced order reference governor } \\ E_{y} & \text { Output error set used by the reduced order reference governor } \\ \xi & \text { Design parameter defined in output error set }\end{array}$

* Graduate Researcher, Email: yetian@umich.edu

†Professor, Email: ilya@umich.edu. This work has been supported in part by the National Science Foundation, Award Number 1130160. 
$y_{r} \quad$ Reduced order system output

Subscripts

$d \quad$ State space representation of discrete-time system

aug System with augmented states

$s d \quad$ Slow subsystem of discrete-time system after fast-slow decomposition

$f d \quad$ Fast subsystem of discrete-time system after fast-slow decomposition

\section{Introduction}

This paper considers the application of reference governors to aircraft engine control. The reference governors are add-on schemes that augment nominally designed controllers to enforce the constraints, see e.g., References ${ }^{3,9}$ and citations therein. The reference governor is nominally inactive and minimally modifies the commands passed to the closed-loop system to ensure that the imposed state and control constraints are satisfied. This modification is performed based on the prediction into the future of the closed-loop system response.

In applying the reference governor to practical systems such as aircraft gas turbine engines, there are several challenges. These challenges include handling situations where satisfying all constraints at once is infeasible, and situations where the system model is high order thereby leading to a high computational complexity of the solution. Recent papers ${ }^{5-7}$ have addressed these challenges. Prioritized reference governor design can enforce hard constraints and soft constraints in the order of their priority. Slight violation of the soft constraints is permitted (if all constraints cannot be strictly met) with less violation for higher priority soft constraints. This is accomplished by introducing and differentially weighting in the cost appropriately defined slack variables. Reduced order reference governor design facilitates rigorous enforcement of the constraints based on the low order slow subsystem models, while accounting for the contributions of both slow states, fast states and observer errors in the prediction. In this paper, these approaches are exemplified for limit protection in aircraft gas turbine engines.

The aircraft gas turbine engine control system must enforce a variety of constraints such as surge avoidance, over-speed and over-temperature limits, combustion lean blowout limit, actuator magnitude and rate limits, etc. ${ }^{4,13}$ Furthermore, the aircraft gas turbine engine models can be high order ${ }^{12}$ even though for control design their order is often reduced ${ }^{4}$ by treating the dynamics of flows and pressures as being faster than the dynamics of the rotational speeds. In Reference ${ }^{10}$ the application of the robust reference governor to aircraft gas turbine engine control based on low order models has been considered. In this paper we exemplify the designs enabled by recent advances in References ${ }^{5,6}$ which include prioritized constraint handling and the treatment based on the high order model via the reduced order reference governor.

In principle, the reference governor is similar to the classical topping governor ${ }^{4}$ used already in aircraft engine control applications. Its main difference with the topping governor is that it accounts for the modelbased prediction of the engine dynamic response to determine command modifications necessary to enforce the full set of engine constraints. The reference governor is also a different strategy from Model Predictive Control (MPC) that has been applied to gas turbine engine control previously, see e.g., References. ${ }^{2,11}$ While the reference governor is a variant of a predictive controller, the main advantages of the reference governor are in augmenting a nominal controller (which can be designed by a variety of linear and nonlinear control design techniques) rather than replacing it with a Model Predictive Controller, rigorously guaranteeing recursive feasibility under command changes, finite-time convergence properties for constant, nearly constant and slowly varying commands, and in simpler computations and software structure.

For the simulation case study in this paper, we use the linear engine model published by Skogestad and Postlethwaite. $^{12}$ The engine under consideration is a Rolls-Royce 2-spool reheated turbofan Spey engine. The model represents the engine dynamics at $87 \%$ of maximum thrust and sea level static conditions.

The paper is organized as follows. Details of the linear model and nominal control design are given in Section II. Constraints are discussed in Section III. In Section IV we review the prioritized and reduced order reference governor design procedures developed in References. ${ }^{5-7}$ In Section $\mathrm{V}$ we consider several case studies where the use of prioritized reference governor and reduced order reference governor is illustrated. Concluding remarks are made in Section VI. 


\section{Aircraft Engine Model}

Our case studies are based on the Spey engine model, ${ }^{12}$

$$
\begin{aligned}
& \dot{x}=A x+B u, \\
& y=C x+D u .
\end{aligned}
$$

The model has 18 states (15 engine states and 3 actuator states), and three control inputs,

$$
u=\left[\begin{array}{c}
W F E \\
A J \\
I G V
\end{array}\right]
$$

where $W F E$ is the fuel flow, $A J$ is the setting for the variable area nozzle, and $I G V$ is the variable angle setting for inlet guide vanes. The model has three outputs,

$$
y=\left[\begin{array}{c}
O P R 1 \\
L P E M N \\
N H
\end{array}\right]
$$

The engine thrust is correlated with the first output (OPR1), which is defined as the ratio of the HP compressor outlet pressure to engine inlet pressure. The second output is the LP compressor exit Mach number measurement ( LPEMN) which is indicative of and used in enforcing the LP compressor surge margin constraint. The third output is HP compressor spool speed $(N H)$; this output is used in defining the over-speed constraint. We do not consider other constraints in this paper as they are not represented in the model published in Reference. ${ }^{12}$

In the model (1)-(2), the thrust-related output $(O P R 1)$ is scaled so that one unit of it represents a $7.5 \%$ of maximum thrust. Similarly, the surge margin-related output $L P E M N$ is scaled so that one unit of it corresponds to $5 \%$ surge margin. Finally, the HP compressor spool speed output $(N H)$ is scaled so that one unit of it corresponds to a $2.2 \%$ of maximum value. The inputs ( $W F E, A J, I G V)$ are scaled so that one unit corresponds to $10 \%$ of each of their respective range.

The nominal controller is based on the LQ tracking design. Specifically, we augment an error integrator to the system with the state $x_{i}$ and use the input WFE to control the output OPR1 to the set-point $r_{O P R 1}$. Then the augmented system takes the form,

$$
\begin{gathered}
\dot{x}_{a u g}=\tilde{A} x_{a u g}+\tilde{B}_{W F E} u_{W F E}+\tilde{B}_{A J, I G V} u_{A J, I G V}+\left[\begin{array}{c}
0_{n 1} \\
-r_{O P R 1}
\end{array}\right], \\
y_{\text {thrust }}=\left[C_{\text {thrust }}, 0\right] x_{a u g}+D(1,:) u,
\end{gathered}
$$

where

$$
x_{a u g}=\left[\begin{array}{c}
x \\
x_{i}
\end{array}\right]
$$

$\tilde{A}=\left[\begin{array}{cc}A & 0_{n 1} \\ C_{\text {thrust }} & 0\end{array}\right], \tilde{B}_{W F E}=\left[\begin{array}{c}B(:, 1) \\ D(1,1)\end{array}\right], \tilde{B}_{A J, I G V}=\left[\begin{array}{c}B(:, 2: 3) \\ D(1,2: 3)\end{array}\right], \tilde{u}_{A J, I G V}=\left[\begin{array}{c}A J \\ I G V\end{array}\right], C_{\text {thrust }}=C(1,:)$.

The fuel flow $(W F E)$ nominal controller is of the form,

$$
u_{W F E}=-K_{1} x-K_{2} x_{i},
$$

where the gains $K_{1}$ and $K_{2}$ are obtained on the basis of the linear quadratic theory applied to $\tilde{A}, \tilde{B}_{W F E}$ and weights $Q$ and $R$ defined by

$$
Q=\left[\begin{array}{cc}
0_{n n} & 0_{n 1} \\
0_{1 n} & 1
\end{array}\right], \quad R=1,
$$


and where $n$ is the dimensionality of $x$.

With the LQ WFE controller, the closed loop system becomes,

$$
\begin{aligned}
& \dot{x}_{a u g}=\left(\tilde{A}-\tilde{B}_{W F E}\left[K_{1}, K_{2}\right]\right) x_{a u g}+\left[\begin{array}{cc}
0_{n 1} & B(:, 2: 3) \\
-1 & D(1,2: 3)
\end{array}\right]\left[\begin{array}{c}
r_{O P R 1} \\
A J \\
I G V
\end{array}\right], \\
& y=\left[C-D(:, 1) K_{1},-D(:, 1) K_{2}\right] x_{a u g}+\left[0_{31}, D(:, 2: 3)\right]\left[\begin{array}{c}
r_{O P R 1} \\
A J \\
I G V
\end{array}\right] .
\end{aligned}
$$

In the application of the reference governor, we replace the commanded

$$
r(t)=\left[\begin{array}{c}
r_{O P R 1} \\
A J \\
I G V
\end{array}\right]
$$

by the reference governor output,

$$
v(t)=\left[\begin{array}{c}
v_{O P R 1} \\
v_{A J} \\
v_{I G V}
\end{array}\right] .
$$

The modified set-points, $v(t)$, may deviate from $r(t)$ if the danger of constraint violation exists.

Converting the continuous-time closed-loop system model to discrete-time with the sampling period of $0.01 \mathrm{sec}$ yields

$$
\begin{gathered}
x_{a u g}(t+1)=A_{d} x_{a u g}(t)+B_{d} v(t), \\
y(t)=C_{d} x_{a u g}(t)+D_{d} v(t),
\end{gathered}
$$

where $A_{d}$ is a 19 by 19 matrix ( 15 engine states, 3 actuator states and 1 controller state), $B_{d}$ is a 19 by 3 matrix ( 3 reference inputs), $C_{d}$ is a 3 by 19 matrix ( 3 outputs), and $D_{d}$ is a 3 by 1 zero matrix.

\section{Constraints}

We consider the following set of constraints. To ensure that an adequate surge margin is maintained, we impose a lower limit on $L P E M N$ of the form,

$$
L P E M N_{\text {min }} \leq L P E M N .
$$

To prevent over-speed conditions, we impose an upper limit on $N H$ of the form,

$$
N H \leq N H_{\max } .
$$

These constraints can be expressed in the following form:

$$
y(t) \in Y, Y=\{y: H y \leq h\},
$$

where

$$
y=\left[\begin{array}{c}
O P R 1 \\
L P E M N \\
N H
\end{array}\right], \quad H=\left[\begin{array}{ccc}
0 & -1 & 0 \\
0 & 0 & 1
\end{array}\right], \quad h=\left[\begin{array}{c}
-L P E M N_{\min } \\
N H_{\max }
\end{array}\right] .
$$

Finally, we consider another constraint which is to avoid large deviations of the output of the reference governor, $v(t)$, from the original command, $r(t)$, i.e.,

$$
-\Delta_{1} \leq v(t)-r(t) \leq \Delta_{2},
$$


where

$$
r(t)=\left[\begin{array}{c}
r_{O P R 1} \\
A J \\
I G V
\end{array}\right], v(t)=\left[\begin{array}{c}
v_{O P R 1} \\
v_{A J} \\
v_{I G V}
\end{array}\right],
$$

and the bounds $\Delta_{1}, \Delta_{2}$ are given.

We note that the constraint (15) is unusual and is not handled by the conventional reference governor theory. In applications, imposing this constraint can be useful to ensure that the reference governor does not create large modifications, however, with no information on future values of $r(t)$, feasibility, if this constraint is handled as a hard constraint, cannot be guaranteed. This constraint can, however, be treated more easily by a prioritized reference governor as we discuss next.

\section{Prioritized and reduced order reference governors}

The reference governor schematics are shown in Figure 1. The reference governor modifies $O P R 1$ as well as $A J$ and $I G V$ settings.

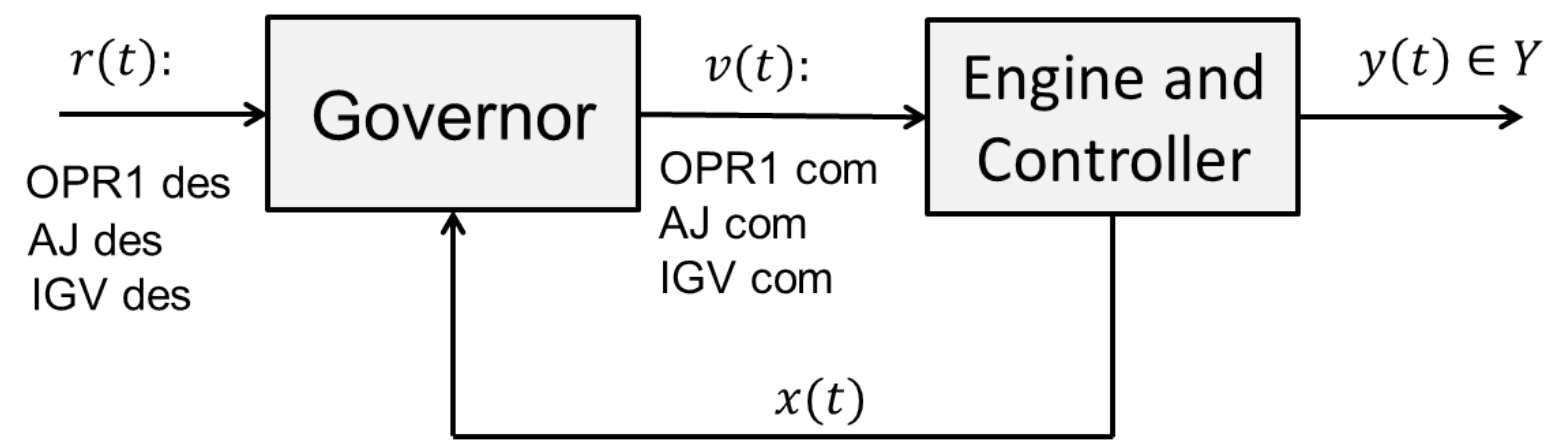

Figure 1: The system architecture. The commands with subscript des and $r(t)$ represent the original commands, the ones with subscript com and $v(t)$ represent modified commands with the reference governor. The reference governor enforces constraints $y(t) \in Y$ using state estimate, $x(t)$.

\section{A. Reference governor}

The conventional reference governor (based on command governor strategy ${ }^{1}$ ) is based on determining $v(t)$ via a solution of the following optimization problem:

$$
\text { minimize }(v(t)-r(t))^{T} Q(v(t)-r(t)),
$$

subject to

$$
(v(t), x(t)) \in \tilde{O}_{\infty},
$$

where $Q=Q^{T}>0$ and $\tilde{O}_{\infty}$ is the maximum output admissible set, ${ }^{3}$ i.e., the set of all constant commands and closed-loop system states such that the ensuing response satisfies the imposed constraints. For the polyhedral-type constraints described in Section III, and under additional, reasonable assumptions, $\tilde{O}_{\infty}$ is a polytope defined by a finite number of linear inequalities.

\section{B. Prioritized reference governor}

In the Prioritized Reference Governor (PRG) approach, ${ }^{5}$ some of the hard constraints are replaced by soft constraints. For instance, the hard output constraints in (14) can be replaced by soft constraints

$$
H y \leq h+\epsilon_{1},
$$


where $\epsilon_{1} \geq 0$ is a vector of slack variables. A similar strategy can be pursued with respect to the constraint (15) by replacing it with a soft constraint

$$
-\Delta_{1}-\epsilon_{2} \leq v(t)-r(t) \leq \Delta_{2}+\epsilon_{2},
$$

where $\epsilon_{2} \geq 0$ is a vector of slack variables. We define

$$
\epsilon=\left[\begin{array}{l}
\epsilon_{1} \\
\epsilon_{2}
\end{array}\right] .
$$

In the PRG design, ${ }^{5}$ the closed loop system is extended with the slack variable vector as follows

$$
\begin{gathered}
x_{a u g}(t+1)=A_{d} x_{a u g}(t)+\left[\begin{array}{ll}
B_{d} & 0
\end{array}\right]\left[\begin{array}{l}
v(t) \\
\epsilon(t)
\end{array}\right], \\
y^{\prime}(t)=\left[\begin{array}{l}
y \\
\epsilon
\end{array}\right]=\left[\begin{array}{c}
C_{d} \\
0
\end{array}\right] x_{a u g}(t)+\left[\begin{array}{cc}
D_{d} & 0 \\
0 & I
\end{array}\right]\left[\begin{array}{l}
v(t) \\
\epsilon(t)
\end{array}\right],
\end{gathered}
$$

and the constraint set $Y$ is replaced by $Y^{\prime}$ where

$$
y^{\prime} \in Y^{\prime}=\left\{(y, \epsilon): \epsilon=\left(\epsilon_{1}, \epsilon_{2}\right), H y \leq h+\epsilon_{1}, \epsilon \geq 0\right\} .
$$

With $\tilde{v}(t)$ defined as:

$$
\tilde{v}(t)=\left[\begin{array}{l}
v(t) \\
\epsilon(t)
\end{array}\right],
$$

and $\tilde{O}_{\infty}^{\prime}$ (or its subset satisfying appropriate assumptions ${ }^{3}$ ) defined for the augmented system, the PRG governs the augmented signal based on the minimization of the cost,

$$
\text { minimize }(r(t)-v(t))^{T} R_{1}(r(t)-v(t))+\epsilon^{T} R_{2} \epsilon \text { w.r.t } v(t), \epsilon,
$$

subject to the constraints,

$$
(\tilde{v}(t), x(t)) \in \tilde{O}_{\infty}^{\prime}, \quad-\Delta_{1}-\epsilon_{2} \leq v(t)-r(t) \leq \Delta_{2}+\epsilon_{2}, \tilde{v}=\left[v^{\mathrm{T}}, \epsilon^{\mathrm{T}}\right]^{\mathrm{T}}, \epsilon=\left[\epsilon_{1}^{\mathrm{T}}, \epsilon_{2}^{\mathrm{T}}\right]^{\mathrm{T}},
$$

where $R_{1}=R_{1}^{\mathrm{T}}>0, R_{2}=R_{2}^{\mathrm{T}}>0$, are weighting matrices. This optimization problem is a quadratic programming problem with linear inequality constraints that can be easily handled by existing quadratic programming solvers.

The weight matrix $R_{2}$ can be defined to give different priorities to different constraints. For instance, if $R_{2}$ is diagonal, diagonal entries corresponding to higher priority constraints can be made larger.

To enforce some constraints as hard the corresponding elements of $\epsilon_{1}$ or $\epsilon_{2}$ can be constrained to zero. Alternatively, the corresponding constraints do not have to be relaxed by the slack variables from the start. ${ }^{6}$

To provide further insight, we note that based on the definition of the maximum output admissible set (and ignoring some technicalities), the minimization of the cost (25) is basically performed subject to constraints

$$
\begin{gathered}
-\Delta_{1}-\epsilon_{2} \leq v(t)-r(t) \leq \Delta_{2}+\epsilon_{2}, \\
H y(t+k \mid t, x(t), v(t)) \leq h+\epsilon_{1}, \forall k=0,1, \ldots, t^{*}, \\
\epsilon_{1} \geq 0, \epsilon_{2} \geq 0,
\end{gathered}
$$

where $y(t+k \mid t, x(t), v(t))$ denotes the output prediction at the time instant $t+k$ given the state $x(t)$ at time $t$, command $v(t)$ (assumed constant over the prediction horizon, i.e., $v(t+k)=v(t), k=0, \cdots, t^{*}$ ), and $t^{*}$ is the finite determination index of the maximum output admissible set. ${ }^{3}$

We note that the use of quadratic penalty functions on the slack variables has certain disadvantages, e.g., soft constraints can be slightly violated by the solution even if a feasible solution that strictly satisfies these constraints exists. If such violations are undesirable (i.e., soft constraints have to be strictly satisfied if possible), one can check for solutions of problem (25)-(26) with slack variables constrained to 0 and progressively relaxed, with highest priority constraints relaxed last. Another approach is to replace the quadratic penalty by linear (e.g., 1 or $\infty$ norm based) penalty with sufficiently large penalty weights. ${ }^{8}$ 


\section{Reduced order prioritized reference governor}

For the development of a reduced order reference governor we consider a state transformation under which the closed-loop dynamic model decomposes into slow and fast subsystems ${ }^{6}$ so that the governing closed-loop equations are given in discrete-time by,

$$
\begin{gathered}
{\left[\begin{array}{l}
x_{s}(t+1) \\
x_{f}(t+1)
\end{array}\right]=\left[\begin{array}{cc}
A_{s d} & 0 \\
0 & A_{f d}
\end{array}\right]\left[\begin{array}{l}
x_{s}(t) \\
x_{f}(t)
\end{array}\right]+\left[\begin{array}{l}
B_{s d} \\
B_{f d}
\end{array}\right] v(t),} \\
y(t)=\left[\begin{array}{ll}
C_{s d} & C_{f d}
\end{array}\right]\left[\begin{array}{l}
x_{s}(t) \\
x_{f}(t)
\end{array}\right]+D_{d} v(t),
\end{gathered}
$$

where $x_{s}(t) \in R^{n_{s}}$ and $x_{f}(t) \in R^{n_{f}}$ are the slow and fast state vectors. If it is assumed that the matrices $A_{s d}$ and $A_{f d}$ are Schur and the eigenvalues of $A_{s d}$ are faster than those of $A_{s f}$.

The reduced order reference governor is designed based on the reduced order system model,

$$
\begin{gathered}
x_{s}(t+1)=A_{s d} x_{s}(t)+B_{s d} v(t), \\
y_{r}(t)=C_{s d} x_{s}(t)+\left(C_{f d} \Gamma_{f}+D_{d}\right) v(t) .
\end{gathered}
$$

where

$$
\Gamma_{f}=\left(I-A_{f d}\right)^{-1} B_{f d},
$$

and $y_{r}$ is the system output that reflects the contributions of the slow states and steady-state contributions of fast states.

The reduced order reference governor is constructed to satisfy the tightened constraint on $y_{r}$ and an additional constraint on $\Delta v(t)=v(t)-v(t-1)$ which ensures that command changes do not cause excessive deviations of fast states from their steady-state values:

$$
\begin{aligned}
& y_{r}(t+k \mid t) \in Y \sim E_{y}, \forall k \geq 0, \\
& -A_{f d} \Gamma_{f} \Delta v(t) \in E_{x} \sim A_{f d} E_{x},
\end{aligned}
$$

where the errors sets $E_{y}$ and $E_{x}$ are design parameters. The error set $E_{y}$ is defined as

$$
E_{y}=\varepsilon Y,
$$

where $0<\varepsilon<1$ is a design parameter. The error set $E_{x}$ is an $A_{f d}$-contractive set that can be computed as the maximum output admissible set corresponding to a scaled $A_{f d}$ matrix and $E_{y}$ as the output constraint set, ${ }^{6}$

$$
E_{x}=O_{\infty}\left(\frac{1}{q} A_{f d}, C_{f d}, E_{y}\right)
$$

where the contraction parameter is $0 \leq q<1$.

For the development of the prioritized reduced order reference governor, we replace (30)-(31) with

$$
\begin{gathered}
{\left[\begin{array}{l}
x_{s}(t+1) \\
x_{f}(t+1)
\end{array}\right]=\left[\begin{array}{cc}
A_{s d} & 0 \\
0 & A_{f d}
\end{array}\right]\left[\begin{array}{l}
x_{s}(t) \\
x_{f}(t)
\end{array}\right]+\left[\begin{array}{cc}
B_{s d} & 0 \\
B_{f d} & 0
\end{array}\right] \tilde{v}(t),} \\
y^{\prime}(t)=\left[\begin{array}{cc}
C_{s d} & C_{f d} \\
0 & 0
\end{array}\right]\left[\begin{array}{l}
x_{s}(t) \\
x_{f}(t)
\end{array}\right]+\left[\begin{array}{cc}
D_{d} & 0 \\
0 & I
\end{array}\right] \tilde{v}(t),
\end{gathered}
$$

where

$$
\tilde{v}(t)=\left[\begin{array}{l}
v(t) \\
\epsilon(t)
\end{array}\right],
$$

and $\epsilon=\left(\epsilon_{1}^{\mathrm{T}}, \epsilon_{2}^{\mathrm{T}}\right)$ is a slack variable as introduced in Section B. The set $Y$ is replaced by $Y^{\prime}$ defined in

$$
Y^{\prime}=\left\{(y, \epsilon): H y \leq h+\epsilon_{1}, \epsilon \geq 0\right\},
$$

and the reduced order reference governor design is applied to this system (39)-(40) augmented with the slack variable $\epsilon$ and with the extended input (41). 


\section{Simulation results}

For the simulations, we choose $L P E M N_{\min }=-1, N H_{\max }=5$ in (12)-(13), and a time-varying set-point command profile given as

$$
r_{O P R 1}(t)=5\left(1-e^{-t}\right), \quad r_{A J}(t)=3, \quad r_{I G V}(t)=2, \quad t>0 .
$$

The command constraints (19) are defined with

$$
\Delta_{1}=\Delta_{2}=\left[\begin{array}{c}
1 \\
1.5 \\
1.5
\end{array}\right] .
$$

We first illustrate the operation of the full order PRG and its flexibility in handling different sets of soft and hard constraints. Three scenarios are considered.

In the first scenario, the surge constraint (12) and speed constraint (13) are treated as soft while the command constraint (19) is treated as hard. We further assign higher priority to the surge constraint than to speed constraint, so that the weight corresponding to the surge constraint is higher than that corresponding to the speed constraint. Then $\epsilon_{2}=0$ (as the command constraints are treated as hard) and the cost (25) is defined with

$$
R_{1}=\operatorname{diag}([100,10,1000]), \quad R_{2}=\operatorname{diag}([100,100,0,0,0]) .
$$

Note that no weighting is applied to $\epsilon_{2}$ since $\epsilon_{2}$ is constrained to zero. This first scenario exemplifies a situation during aircraft emergency maneuvering where the control system accepts some level of risk ${ }^{13}$ in enforcing the surge and speed constraints (as these are often set conservatively to begin with) provided it can ensure adequate thrust response.

In the second scenario we treat the surge constraint as hard and the speed constraint and command constraints as soft. We assign higher priority to speed constraint than to command constraints. Since there are three command constraints, the weight on the slack variable corresponding to $O P R 1$ command is larger than weights corresponding to $A J$ and $I G V$ commands. In this case, we constrain $\epsilon_{1}$ to $\epsilon_{1}=\left[0, \epsilon_{12}\right]$, where $\epsilon_{12}$ is a scalar and treat $\epsilon_{2}$ as a three dimensional vector. The PRG weighting matrices are defined as

$$
R_{1}=\operatorname{diag}([100,10,1000]), \quad R_{2}=\operatorname{diag}([0,10000,1000,100,100]) .
$$

Note that no penalty is applied to the first component of $\epsilon_{1}$ since this component is constrained to be zero.

In the third scenario, the constraint and weight matrix are the same as in the second scenario. However, instead of applying an exponential profile (42) for OPR1, we investigate the response to a step change in $O P R 1$ to the value 5 at 1 second.

The simulation results for these three scenarios are given in Figures 2-7. For the first simulation scenario, the speed constraint is violated, the surge constraint is violated less and the commands passed to the closedloop system ride the constraint boundary. For the second simulation scenario, the surge constraint is strictly enforced, speed constraint is violated slightly while $v_{O P R 1}$ exceeds the bounds. For the third simulation scenario, the surge constraint is strictly enforced, speed constraint is violated slightly while $v_{O P R 1}$ exceeds the bounds and $v_{A J}$ is out of bounds for a short time to accommodate the sudden step change of $O P R 1$.

Finally, we illustrate the operation of the reduced order PRG for the first scenario. The absolute values of the eigenvalues of the continuous-time closed-loop system model are

$$
1.8763,6.2272,6.2272,31.1960,31.2500,44.5930,61.9103,92.7397,92.7397
$$

$$
\text { 154.1014, 154.1014, 158.6060, 189.3077, 189.3077, 246.4429, 246.4429, 287.7015, 287.7015, 327.2601. }
$$

By considering eigenvalues with absolute values above 100 as fast we obtain a system decomposition into a slow subsystem with 9 states and a fast subsystem with 10 states. The reduced order PRG uses $q=0.8$, $\varepsilon=0.02$ (see (37)-(38) where these parameters are defined). The simulation results are given in Figures 8-9. Further model order reduction has been investigated. With the slow subsystem of 3rd order, the responses are not significantly different than the ones in Figures 8-9, with somewhat larger soft constraint violation, see Figures 10-11. 


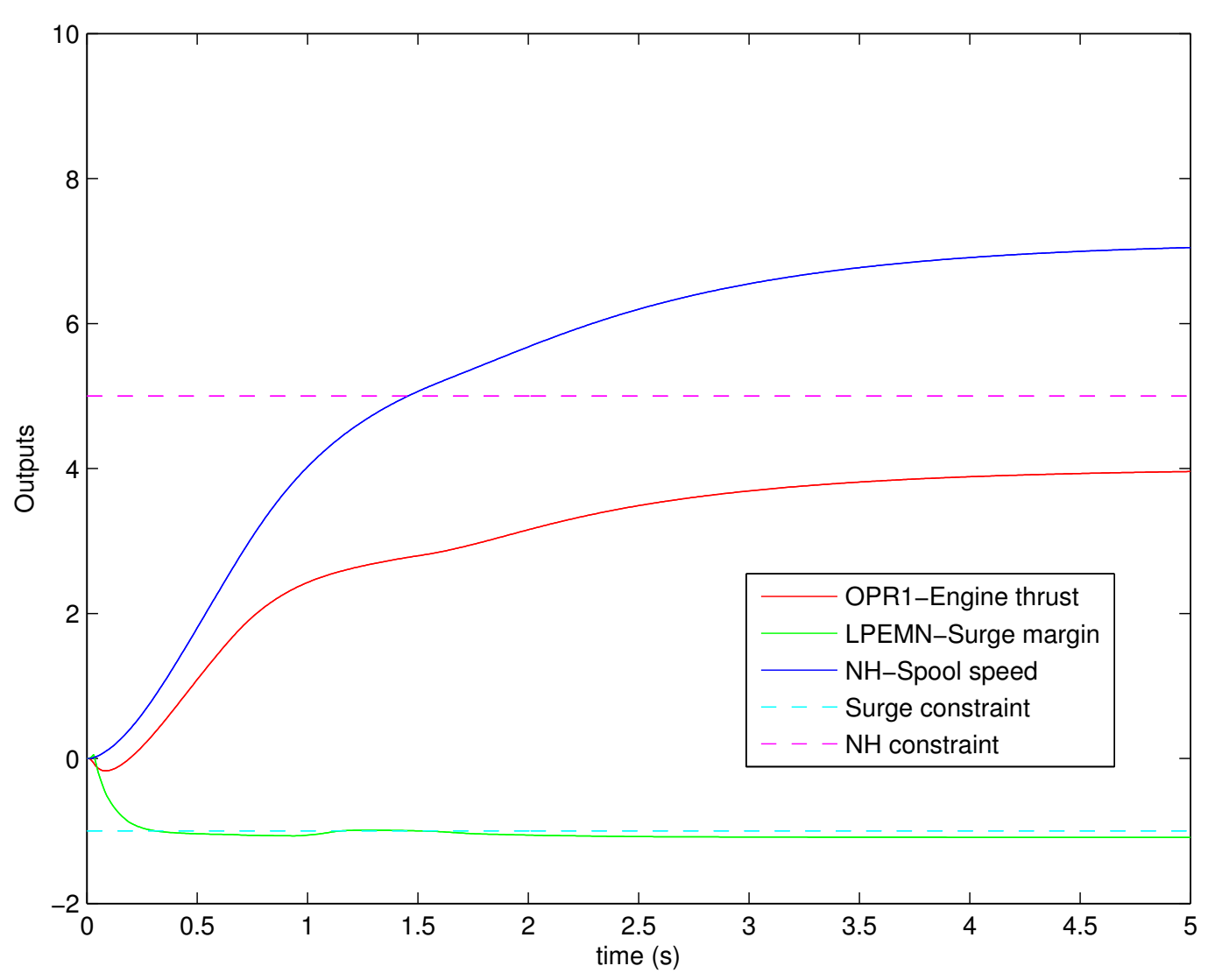

Figure 2: Response with PRG in scenario 1. 

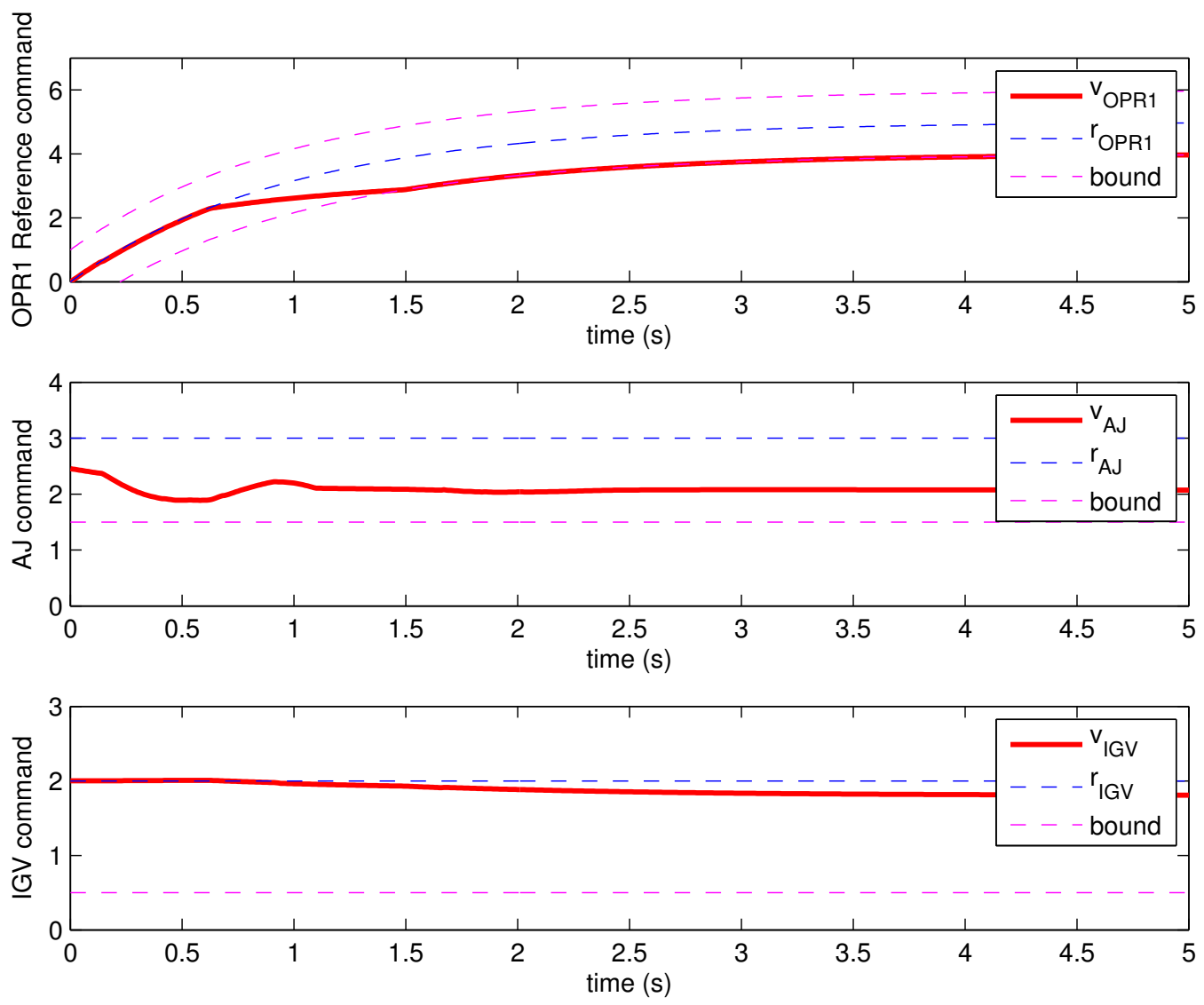

Figure 3: Commands (original and modified) with PRG in scenario 1.

10 of 19 


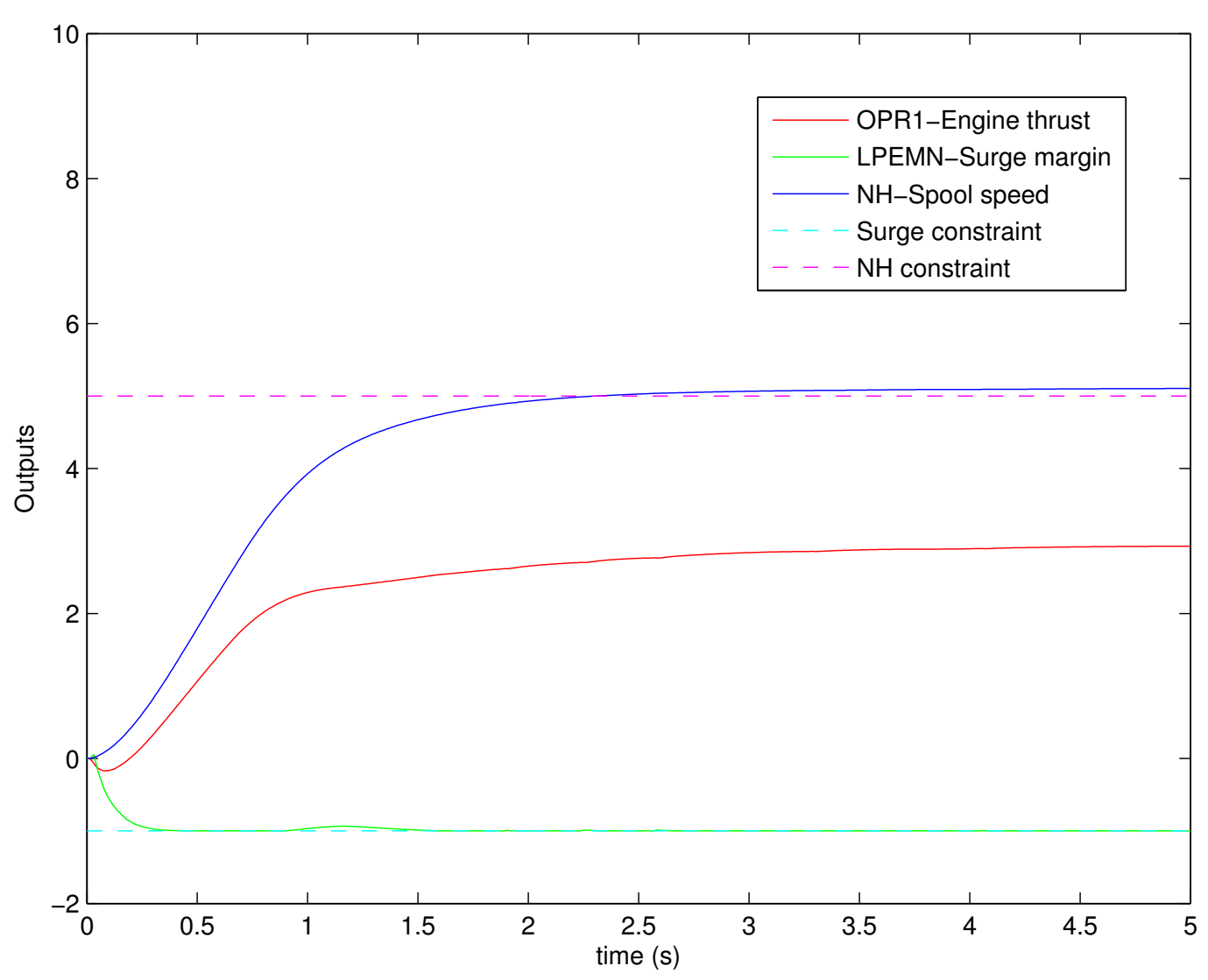

Figure 4: Response with PRG in scenario 2. 

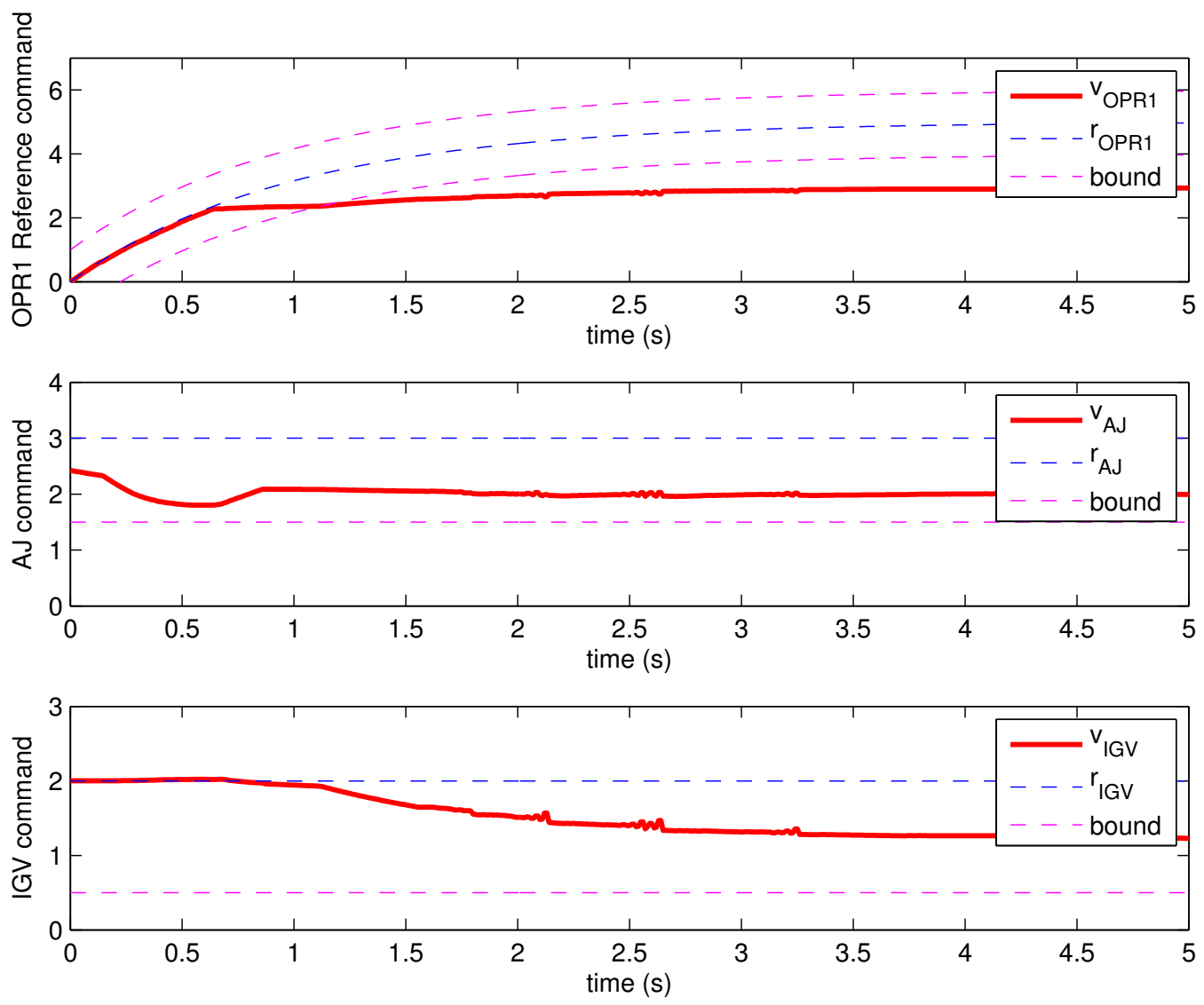

Figure 5: Commands (original and modified) with PRG in scenario 2. 


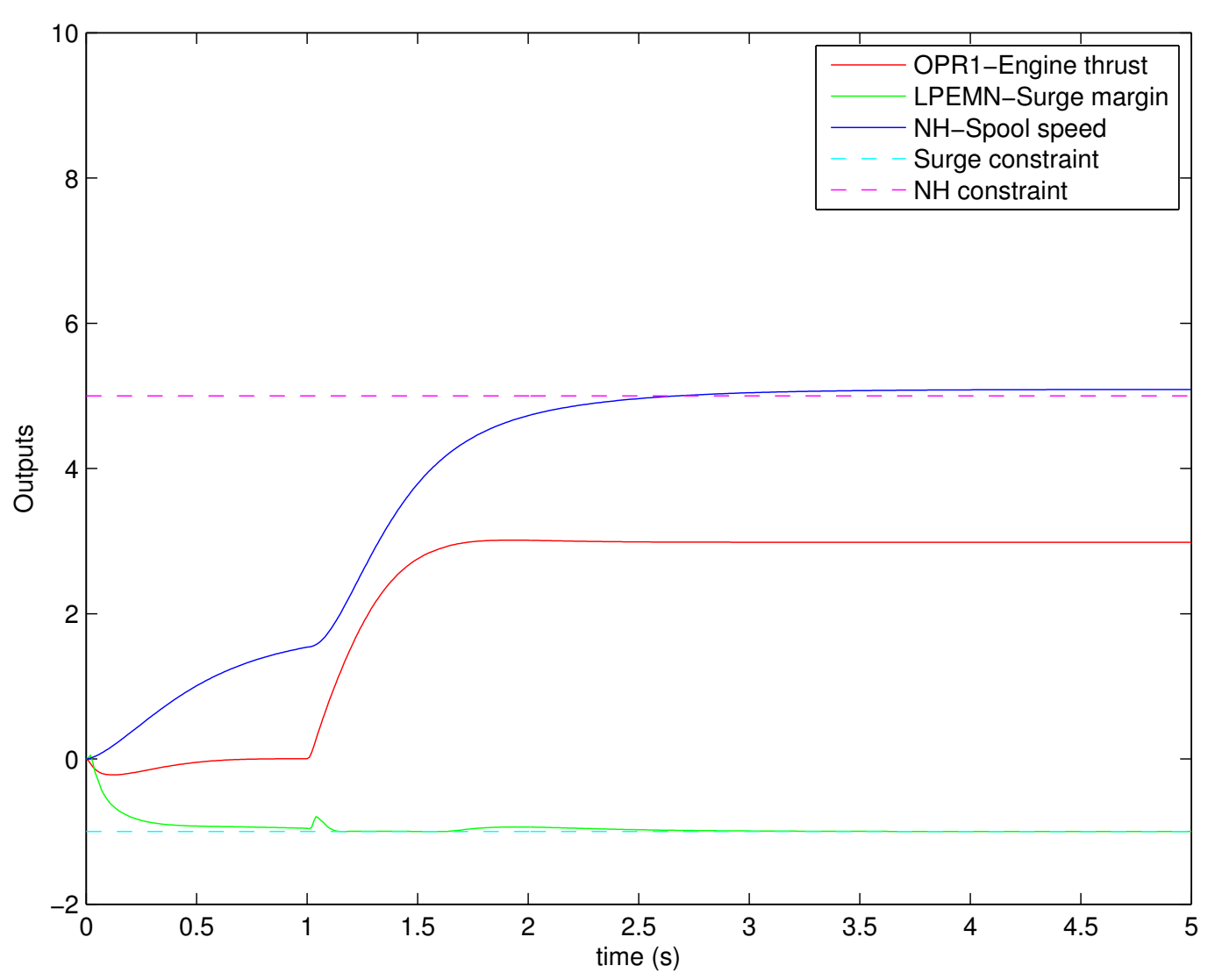

Figure 6: Response with PRG in scenario 3. 

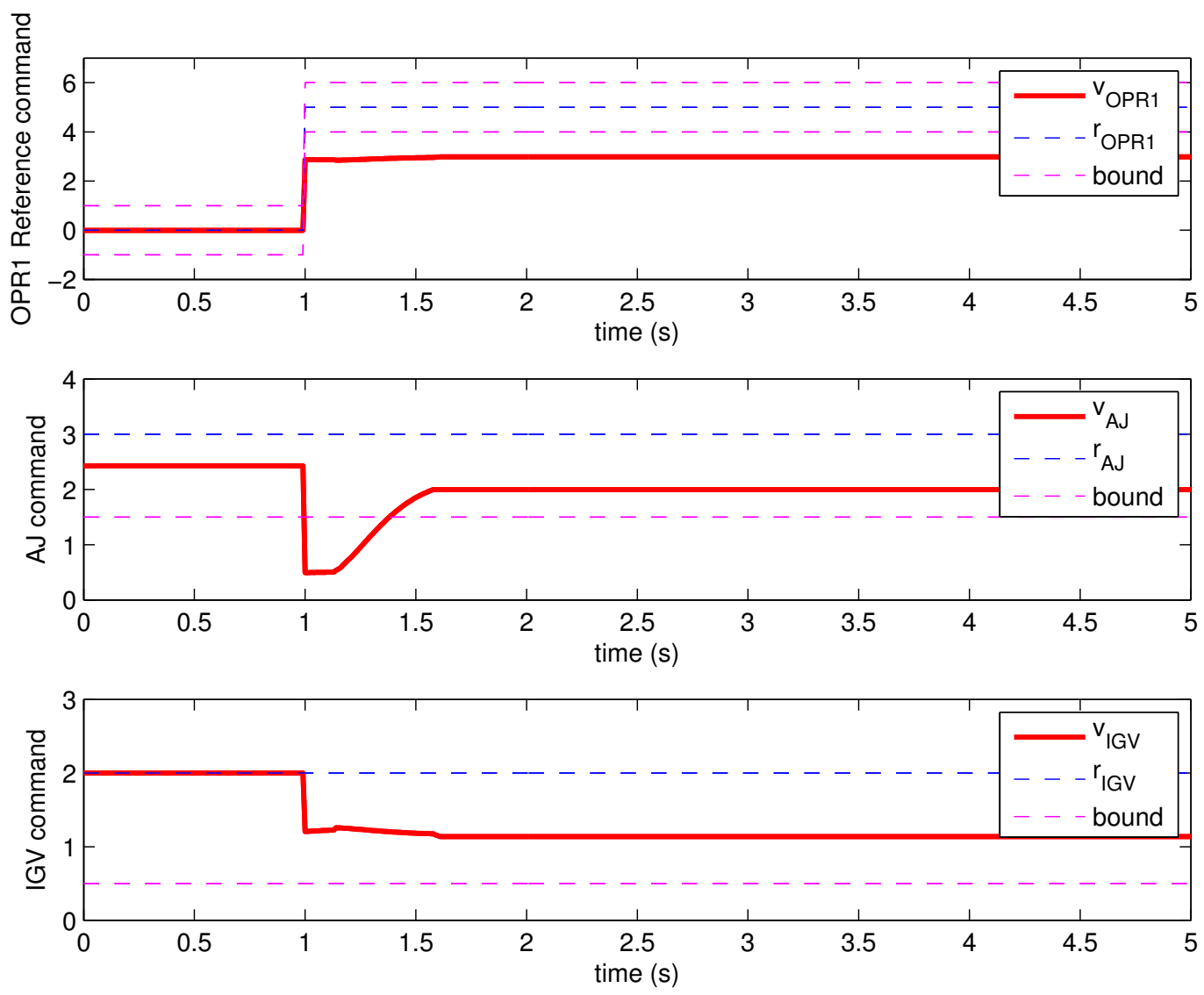

Figure 7: Commands (original and modified) with PRG in scenario 3. 


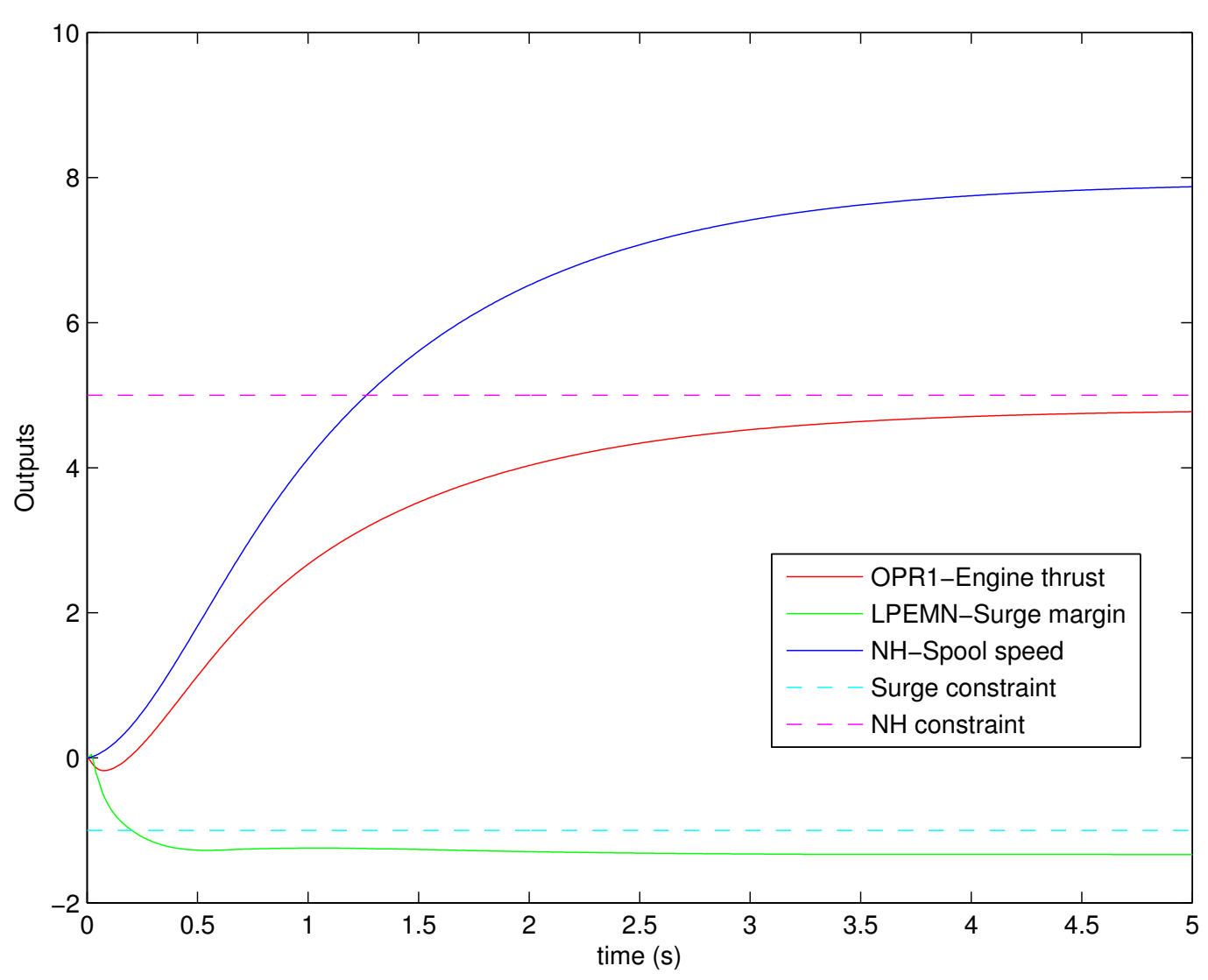

Figure 8: Responses with the reduced order PRG in scenario 1.

15 of 19 

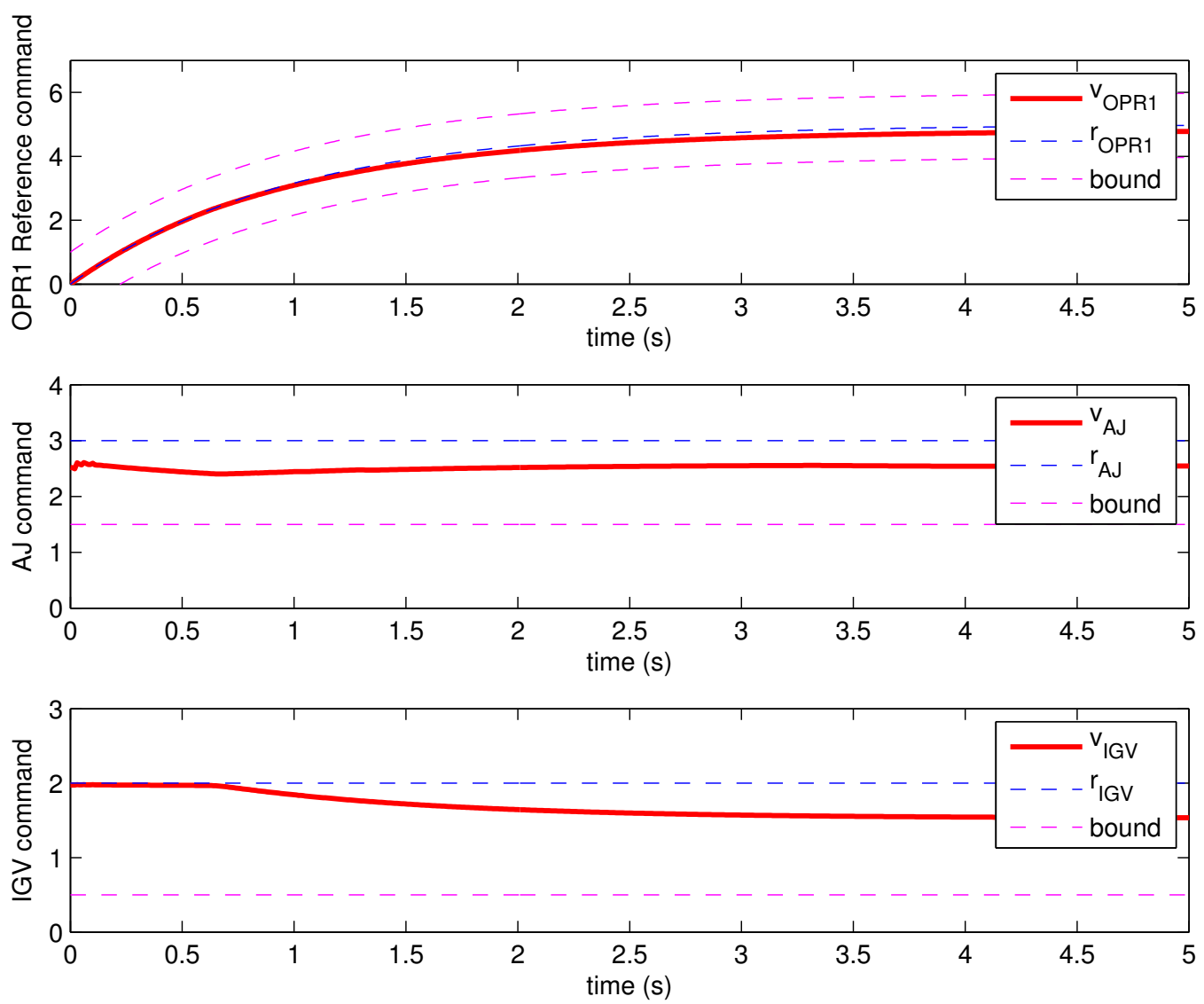

Figure 9: Commands (original and modified) with the reduced order PRG in scenario 1. 


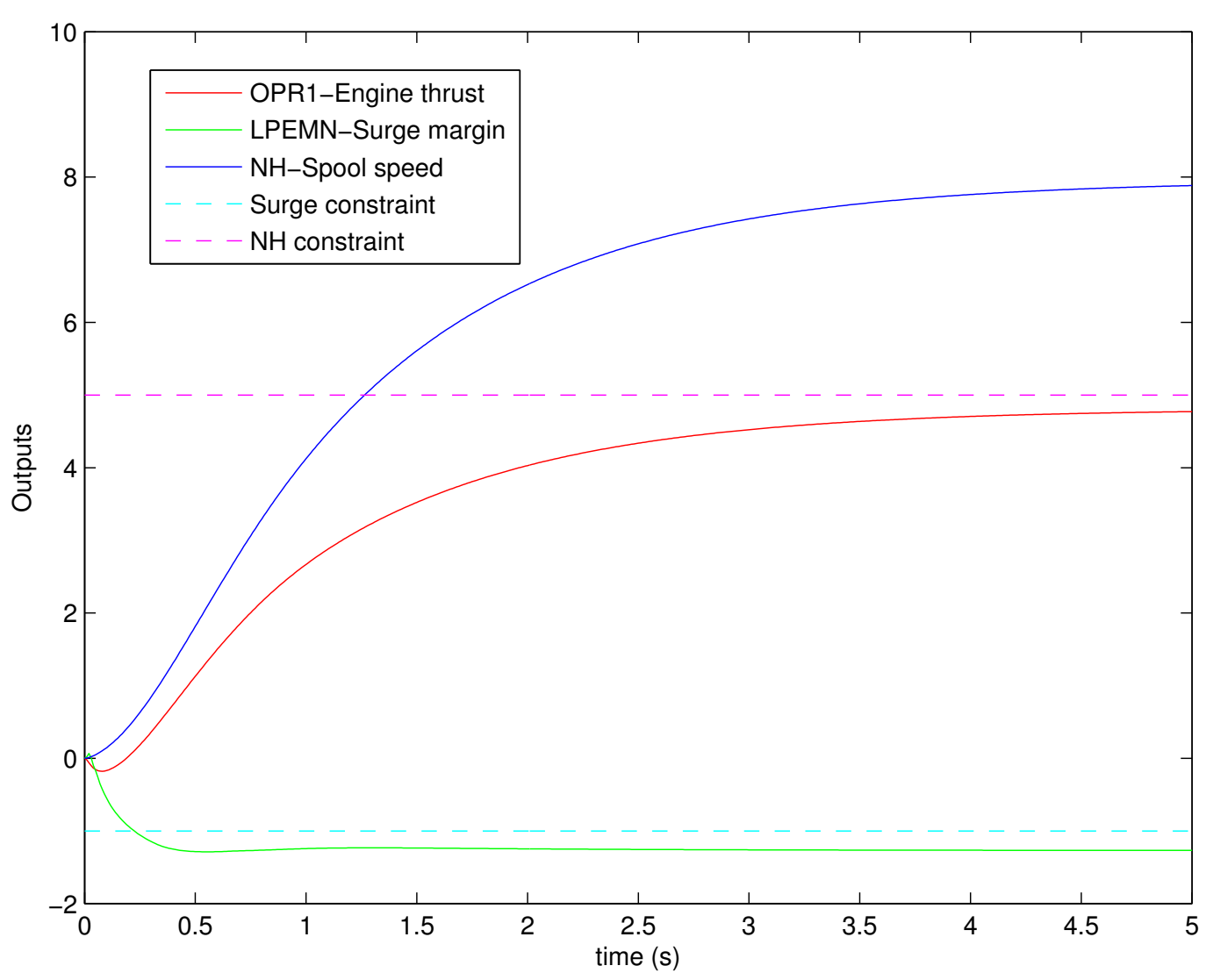

Figure 10: Responses with the reduced order PRG (subsystem of 3rd order) in scenario 1. 

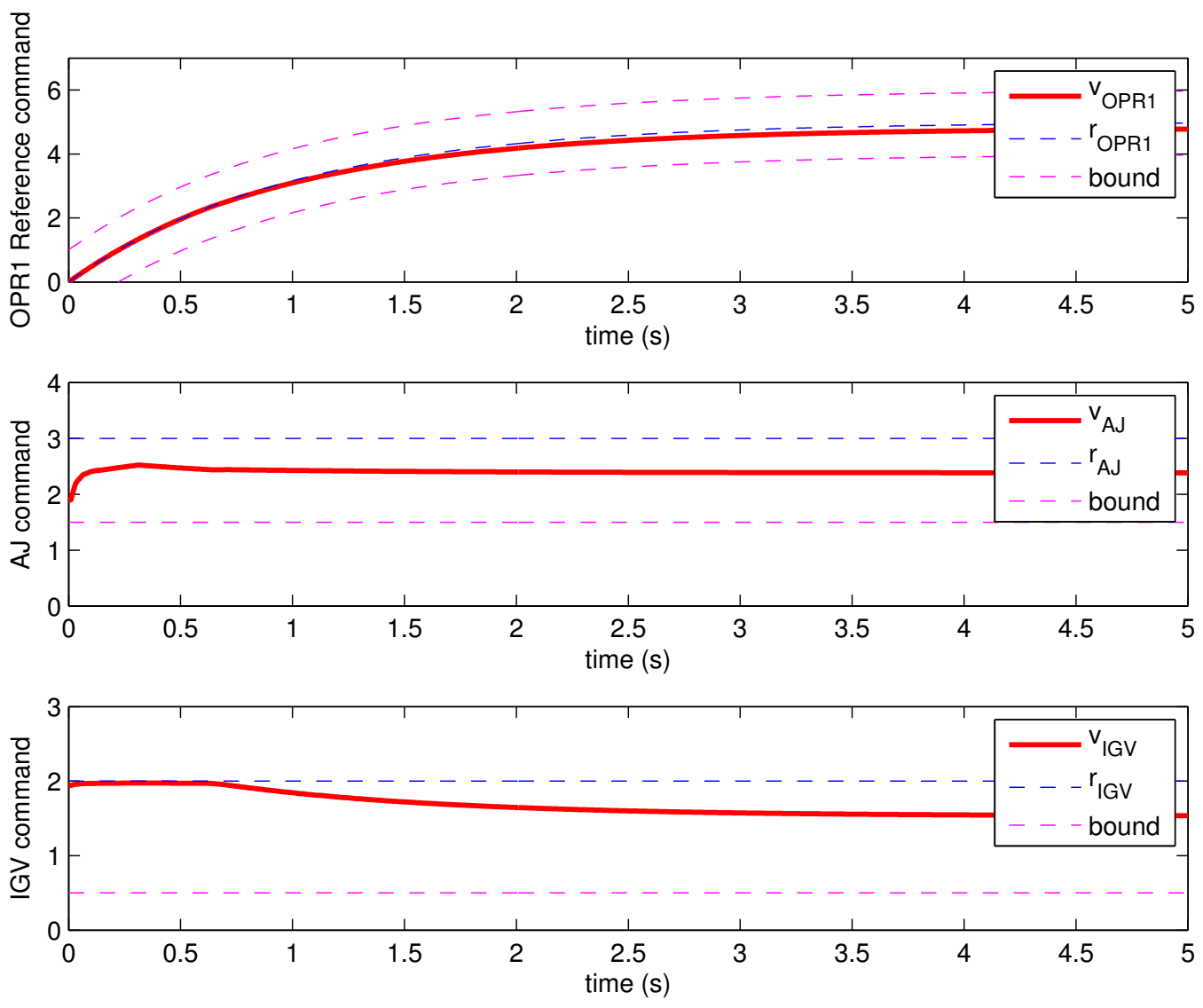

Figure 11: Commands (original and modified) with the reduced order PRG (subsystem of 3rd order) in scenario 1. 


\section{Concluding remarks}

The paper illustrated the use of recently developed prioritized and reduced order reference governors for constraint handling in gas turbine engines. The prioritized reference governor addresses the situations when satisfying all the constraints at once becomes infeasible. It treats some of the constraints as soft (this selection of the soft constraints can be situation-dependent) and minimizes their violation reflective of the specified constraint priority. The reduced order reference governor rigorously enforces the constraints based on a low order model while accounting for the contributions of the omitted states. For the model used in this paper, the full order model had 19 states and we demonstrated that the design of a reduced order prioritized reference governor can be based on the 9 th and 3rd order slow subsystem models. The full state was assumed to be available for implementation of the reference governor schemes in the simulations in this paper. While outside the scope of this paper, the observer estimation errors can also be rigorously handled. ${ }^{6,7}$

\section{References}

${ }^{1}$ A. Bemporad, A. Casavola, and E. Mosca, "Nonlinear control of constrained linear systems via predictive reference management," IEEE Trans. on Autom. Control, vol. 42, pp. 340-349, 1997.

${ }^{2}$ Brunell, B., "Methods and apparatus for Model Predictive Control of aircraft gas turbine engines," U.S. Patent 6823253, 2004.

${ }^{3}$ Gilbert, E., and Kolmanovsky, I.., "Fast reference governors for systems with state and control constraints and disturbance inputs, International Journal of Robust and Nonlinear Control, vol. 9, no. 15, pp. 1117-1141, 1999.

${ }^{4}$ Jaw, L.C., and Mattingly, J.D., Aircraft Engine Controls: Design, System Analysis, and Health Monitoring, AIAA Education Series, 2009.

${ }^{5}$ Kalabic, U., Chitalia, Y., Buckland, J., and Kolmanovsky, I.V., "Prioritization schemes for reference and command governors," Proceedings of 2013 European Control Conference, Zurich, pp. 2734-2739, 2013.

${ }^{6}$ Kalabic, U., Kolmanovsky, I.V., Buckland, J., and Gilbert, E.G., "Reduced order reference governor," Proceedings of 51 st IEEE Conference on Decision and Control, pp. 3245-3251, 2012.

${ }^{7}$ Kalabic, U., Kolmanovsky, I.V., and Gilbert, E.G., "Reduced order extended command governor," Automatica, provisionally accepted for publication.

${ }^{8}$ Kerrigan, E. C. and Maciejowski, J. M., Soft constraints and exact penalty functions in Model Predictive Control,Proceedings of UKACC International Conference,2000.

${ }^{9}$ Kolmanovsky, I.V., Kalabic, U., and Gilbert, E.G., "Developments in constrained control using reference governors," Proceedings of the 2012 IFAC Conference on Nonlinear Model Predictive Control (NMPC), Noordwijkerhout, the Netherlands, August $23-27,2012$.

${ }^{10}$ Kolmanovsky, I.V., Jaw, L., Merrill, W., and H.-T. Tran, "Robust control and limit protection in aircraft gas turbine engines," Proceedings of the 2012 IEEE Multi-conference on Systems and Control, Dubrovnik Palace Hotel, Dubrovnik, Croatia, October 3-5, 2012.

${ }^{11}$ Richter, H., Advanced Control of Turbofan Engines, Springer, 2011.

${ }^{12}$ Skogestad, S., and Postlethwaite, I., Multivariable Feedback Control Analysis and Design, Second Edition, Wiley, August $29,2001$.

${ }^{13}$ Weiss, A., Kolmanovsky, I.V., and Merill, W., "Incorporating risk into control design for emergency operation of turbo-fan engines," Proceedings of Infotech@Aerospace, St. Louis, Missouri, March 29-31, 2011, AIAA Paper AIAA-2011-1591. 